Fugitive Freedom 
The publisher and the University of California Press Foundation gratefully acknowledge the generous support of the Peter Booth Wiley Endowment Fund in History. 


\section{Fugitive Freedom}

THE IMPROBABLE LIVES OF TWO IMPOSTORS IN LATE COLONIAL MEXICO

\section{William B. Taylor}

प UNIVERSITY OF CALIFORNIA PRESS 
University of California Press

Oakland, California

(c) 2021 by William B. Taylor

Library of Congress Cataloging-in-Publication Data

Names: Taylor, William B., author.

Title: Fugitive freedom : the improbable lives of two impostors in late colonial Mexico / William B. Taylor.

Description: Oakland, California : University of California Press, [2021] | Includes bibliographical references and index.

Identifiers: LC C N 2020025987 (print) | LC C 2020025988 (ebook) | IS BN 9780520368569 (cloth) | ISBN 9780520976146 (ebook)

Subjects: LC SH: Aguayo y Herrera, Joseph Lucas, 1747- | Atondo, Juan, 1783?- | Catholic Church-Mexico-History-18th century. | Impostors and imposture-Mexico-18th century. | Church and state-MexicoHistory-18th century. | Mexico-Church history-18th century.

Classification: LC C HV 6761.M 62 A 4872021 (print) | LCC HV 6761.M 62 (ebook) | D D 364.16/33092272-dc23

LC record available at https://lccn.loc.gov/2020025987

LC ebook record available at https://lccn.loc.gov/2020025988

Manufactured in the United States of America

$\begin{array}{llllllllll}30 & 29 & 28 & 27 & 26 & 25 & 24 & 23 & 22 & 21\end{array}$

$\begin{array}{llllllllll}10 & 9 & 8 & 7 & 6 & 5 & 4 & 3 & 2 & 1\end{array}$ 